\title{
CHANGE OF SEED GERMINATION RATE WITH STORAGE TIME OF Santalum album 1. (INDIAN SANDALWOOD) SEEDS
}

\author{
Y.M.M. Gamage ${ }^{1}$, S.M.C.U.P. Subasinghe ${ }^{1}$ and D.S. Hettiarachchi ${ }^{2}$ \\ ${ }^{1}$ Department of Forestry and Environmental Science, University of Sri Jayewardenepura, Sri Lanka. \\ ${ }^{2}$ Wescorp Sandalwood Pty. Ltd., Bibra Lake, WA 6163, Australia. \\ Email: manurigamage@yahoo.com, Tel: +947 72209255
}

\begin{abstract}
Not like other trees species, Indian sandalwood (S. album) nurseries needs more space due to the requirement of spaces for seedling hosts and field hosts in addition to the space required for the sandalwood seedlings. Therefore the number of seedlings that can be raised in a sandalwood nursery is comparatively low. Moreover, sandalwood seeds can be obtained only twice per year and due to those reasons, storage of seeds becomes a necessity. The dried sandalwood seeds are mostly stored in cotton bags. The aim of the current study was to identify the change of germination rate with the storage period under the above mentioned storage method.

For this purpose, seeds collected soon after falling to the ground were stored in cotton bags after preliminary cleaning. Then the seeds were germinated on weekly basis after a pre-treatment with $0.05 \%$ gibberelic acid $\left(\mathrm{GA}_{3}\right)$. Water was used instead of $\mathrm{GA}_{3}$ for the control experiment. As the storage time increased it was observed that the number of seeds germinated decreased. The maximum germination percentage of $>80 \%$ was observed when the storage period was 3 weeks. Germination of $50 \%$ was achieved at the $7^{\text {th }}$ week of storage and the germination rate was zero percent after 28 weeks of storage under the $\mathrm{GA}_{3}$ treatment. The control experiment indicated comparatively a lower germination rate than the treatment with the $\mathrm{GA}_{3}$.
\end{abstract}

\section{INTRODUCTION}

Indian sandalwood (Santalum album) is a commercially and culturally important plant species, known for its fragrant heartwood and oil. The sandalwood oil, known as santalol is highly demanded by the perfume industry due to its sweet fragrance, persistent aroma and the fixative property (Jain et al., 2003). Due to the increase of worldwide demand for sandalwood and the decline of sandalwood natural resource, Indian sandalwood has gained the interest as a plantation crop in Sri Lanka in the recent years.

Propagation of sandalwood is commonly done by seeds, which are produced only once or twice per year from June to September and/or November to February. Therefore storage of sandalwood seeds has to be done especially in large scale plantation establishment. However, the seed viability decreases with storage time which is a common phenomenon for tree seeds. Therefore the present study was initiated to identify the seed germination rate of sandalwood seeds over storage period.

\section{STUDY SITE}

The experiment was carried out in the green house at the Department of Forestry and Environmental Science, University of Sri Jayewardenepura. The average temperature was $30^{\circ} \mathrm{C}$, with the minimum of $27^{\circ} \mathrm{C}$ and the maximum of $32^{\circ} \mathrm{C}$.

\section{MATERIALS AND METHODS}

Sandalwood seeds collected within a 3 day period from a well grown 10-15 years old mother tree in Welimada region, were used for the present study. Readily fallen seeds were collected from the ground on the month of October 2009. Seeds were soaked in water, depulped by

Proceedings of the 15 ${ }^{\text {th }}$ International Forestry and Environment Symposium, 26-27 November 2010.

Published by Department of Forestry and Environmental Science, University of Sri Jayewardenepura, Sri Lanka. 
removing the fleshy fruit part and were air dried to remove the moisture before storing. Dried seeds were then stored in cotton bags (The most accepted storage method) until ready for sowing.

Seeds were sown in seed beds $(45 \times 45 \mathrm{~cm})$ made of bricks. Those were capable of accommodating 100 seeds per bed. Cleaned river sand was used as the germination media that was filled in the seed beds up to a height of $18 \mathrm{~cm}$. Bottom layer was filled with small brick particles sized to about $2.5 \mathrm{~cm}$. In order to avoid the common fungal attacks on sandalwood seeds, the seeds and the germination medium were treated with Captan fungicide ( $12 \mathrm{~g}$ per $10 l$ of water for $2 \mathrm{~m}^{3}$ of area), prior to the sowing. This fungicide was further used once in every two weeks after sowing. Watering was done once per day in the mornings, about $1 l$ per seed bed.

Starting from a storage period of 3 weeks, 100 seeds from the seed storage were treated with $0.05 \%$ gibberelic acid $\left(\mathrm{GA}_{3}\right)$ for $12 \mathrm{hrs}$ prior to sowing and were sown in once a week for until storage period of 12 weeks. From the $12^{\text {th }}$ week onwards the seeds were sown once in two weeks until the storage period of 32 weeks ( 8 months). Instead of gibberelic acid, water was used for the control. Control experiment was done once in 4 weeks starting from the $4^{\text {th }}$ week of seed storing.

Number of seeds germinated after two months was counted as the measurement in the seed germination vs storage experiment.

\section{RESULTS}

The figure 1 illustrates the germination percentage values of sandalwood seeds stored for different time periods.

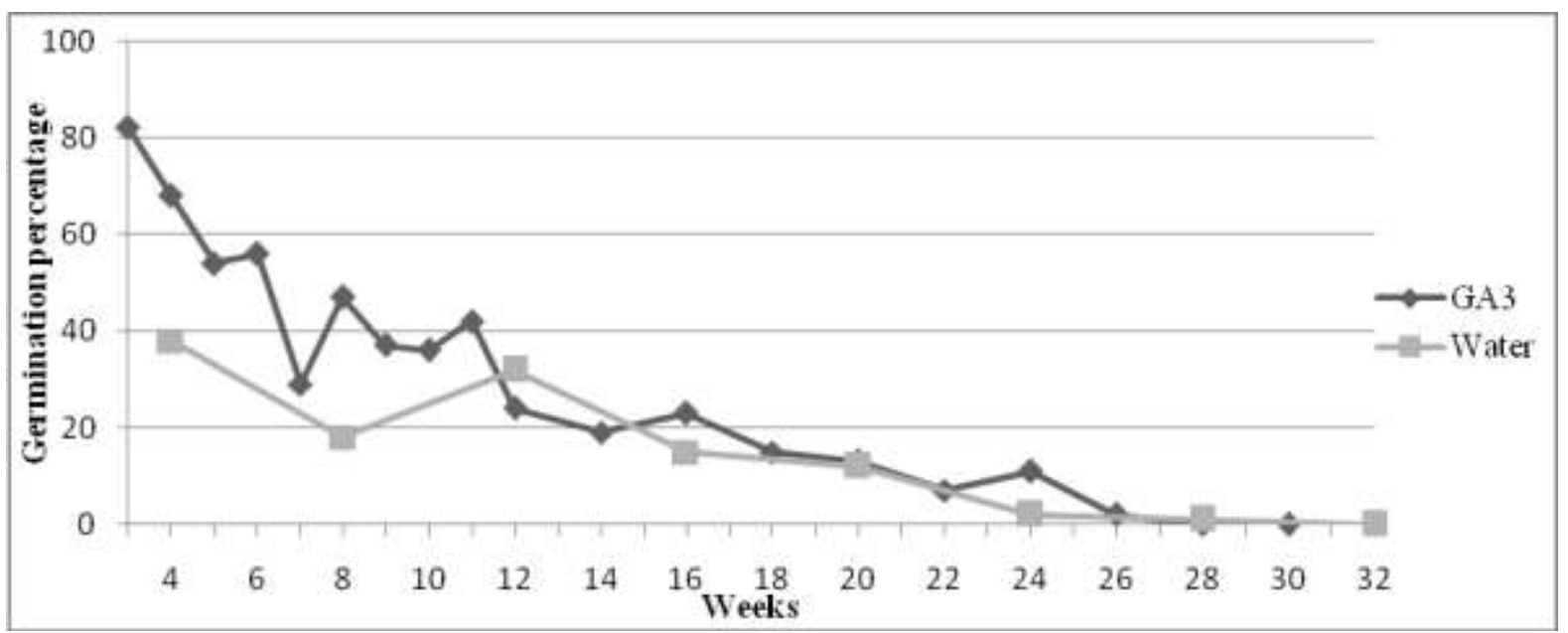

Figure 1: Germination percentage of sandalwood seeds with storage time

According to the figure 1, the number of seeds germinated showed a decreasing germination rate with storage time under both treatments. The maximum germination percentage of $>80 \%$ was observed when the storage period was 3 weeks under the $\mathrm{GA}_{3}$ treatment and the germination rate was less than $40 \%$, when seeds were soaked in water at the same time. Germination of $50 \%$ was achieved at the $7^{\text {th }}$ week of storage and the germination rate was zero percent after 28 weeks of storage under the $\mathrm{GA}_{3}$ treatment. 


\section{DISCUSSION}

The present experiment showed a negative relationship of germination percentage of sandalwood seeds over storage time, which also proved the common phenomenon of tree seeds. The present study showed a germination rate of $50 \%$ with storage period of 7 weeks. However, the viability of sandalwood seeds varies between individual trees (Effendi and Surata, 1993) and also between populations (Brand, 1993; Susila et al., 1995). Furthermore, the age of the mother tree and its location may influence the production of fertile seeds. It was identified that mature trees produce more fertile seeds (Neil, 1990). Further, the germination percentages vary depending on the storage method (Tennakoon et al., 2000).

Although tested in many ways, the vegetative propagation has not been successful for sandalwood (Uniyal et al., 1985). Therefore propagation of sandalwood is commonly done by propagating seeds in nurseries or directly sowing them in the field (Dayal, 1986; Fox, 1989). Therefore it is required to identify methods in order to maintain higher seed germination rates over storage periods for sandalwood seeds.

\section{ACKNOWLEDGEMENT}

Financial support given by the Sadaharitha Plantations Pvt. Ltd. is acknowledged.

\section{REFERENCES}

Brand. J.E. (1993) Phenotype and genotype variation within Santlum album in West Timor. In: Setiadi, S. and Komar, T.E., 2001 